\title{
IABM: Instrumento para Avaliação de Bases de Medidas para Controle Estatístico de Processos de Software
}

\author{
Monalessa Perini Barcellos ${ }^{1,2}$, Ana Regina C. da Rocha ${ }^{1}$, Ricardo de A. Falbo ${ }^{2}$ \\ ${ }^{1}$ COPPE/UFRJ - Universidade Federal do Rio de Janeiro \\ Programa de Engenharia de Sistemas e Computação \\ Caixa Postal 68511 - CEP 21945-970 - Rio de Janeiro, RJ, Brasil \\ ${ }^{2}$ UFES - Universidade Federal do Espírito Santo - Centro Tecnológico \\ Departamento de Informática - CEP 29075-910 - Vitória, ES, Brasil \\ monalessa@inf.ufes.br; darocha@cos.ufrj.br; falbo@inf.ufes.br
}

\begin{abstract}
Resumo. As organizações de software têm mostrado um crescente interesse por programas de melhoria de processos. Na alta maturidade, a melhoria de processos de software envolve a implementação do controle estatístico de processos, que requer que tenham sido definidas medidas e coletados dados úteis a esse contexto. No entanto, essa tem sido uma das principais dificuldades apontadas pela literatura para a realização bem sucedida do controle estatístico de processos. Considerando essa questão, este artigo apresenta um instrumento para avaliação de bases de medidas com o objetivo de auxiliar as organizações de software na preparação e implementação do controle estatístico de processos.
\end{abstract}

\begin{abstract}
Software organizations have increased their interest on software process improvement. In high maturity, software process improvement involves implementation of the statistical process control, which requires measures and data useful to this context. However, this has been one of the main difficulties reported in the literature to statistical process control successful implementation. Considering that issue, this article presents an instrument to evaluate measures repositories aiming to support software organizations in the statistical process control preparation and implementation.
\end{abstract}

\section{Introdução}

O controle estatístico de processos foi originalmente desenvolvido na área de manufatura, visando apoiar a implementação de programas de melhoria contínua em linhas de produção[Lantzy, 1992]. Apesar de sua utilização na melhoria de processos não ser novidade para a indústria em geral, no contexto das organizações de software o controle estatístico pode ser considerado relativamente recente, existindo ainda muitas dúvidas sobre sua aplicação [Card, 2004 ; Card et al., 2008].

As experiências com a realização do controle estatístico de processos nas organizações têm revelado aos pesquisadores e profissionais de empresas um cenário caracterizado por problemas e situações não favoráveis ou que impossibilitam a implantação e realização bem sucedida do controle estatístico de processos [García et al., 2004; Tarhan e Demirors, 2006; Wang et al., 2007; Boffoli et al., 2008; Tarhan e Demirors, 2008]. Nesse contexto destaca-se a não adequação das medidas coletadas 
pelas organizações, o que retarda a realização do controle estatístico de processos, uma vez que, primeiramente, deve ser realizada a adequação das medidas para só então ser possível aplicar as técnicas do controle estatístico [Wheeler e Poling, 1998; Kitchenham et al., 2006; Tarhan e Demirors, 2006; Kitchenham et al., 2007; Curtis et al., 2008; Gou et al., 2009].

Em organizações de alta maturidade, caracterizada nos níveis A e B do MR MPS - Modelo de Referência para Melhoria de Processo de Software Brasileiro [Softex, 2009] e nos níveis 4 e 5 do CMMI - Capability Maturity Model Integration [Chrissis et al., 2006], a aplicação do controle estatístico de processos sucede um programa de medição que foi executado e alimentou uma base organizacional de medidas, sendo desejável que as medidas presentes nessa base sejam adequadas ao controle estatístico de processos. Porém, conforme mencionado anteriormente, de acordo com os relatos da literatura, esse não tem sido um fato comum.

Nesse contexto, este artigo apresenta um Instrumento para Avaliação de Bases de Medidas (IABM) considerando a adequação ao controle estatístico de processos de software, que foi avaliado em três organizações de software e evoluído com base na experiência adquirida e no feedback obtido. Este artigo descreve a evolução dos trabalhos registrados anteriormente em [Barcellos e Rocha, 2008a, b]. O artigo encontra-se assim organizado: na seção 2 é realizada uma breve fundamentação teórica sobre controle estatístico de processos; na seção 3 é descrito o desenvolvimento do IABM; na seção 4 o IABM propriamente dito é apresentado; na seção 5 são discutidos alguns trabalhos correlatos; e, na seção 6 são realizadas as conclusões do artigo.

\section{Controle Estatístico de Processos}

A crescente exigência do mercado por produtos e serviços cada vez melhores tem aumentado o interesse das organizações pela melhoria de processos. Para apoiar a avaliação e melhoria de processos, uma variedade de iniciativas tem proposto frameworks a serem seguidos. Exemplos notáveis são o MR MPS [Softex, 2009], o CMMI [Chrissis et al., 2006], a ISO/IEC 15504 [ISO/IEC, 2003] e a ISO/IEC 12207 [ISO/IEC, 2008]. Em todas essas iniciativas a medição ocupa papel fundamental para a institucionalização de programas de melhoria de processos.

Para utilizar a medição em um contexto de alta maturidade na melhoria de processos de software, novos conceitos e práticas devem ser adicionados aos programas de medição tradicionais, uma vez que a melhoria de processos em alta maturidade requer conhecimento consistente do comportamento dos processos em suas execuções nos projetos da organização, o que é possível através do uso do controle estatístico de processos.

O controle estatístico de processos utiliza um conjunto de ferramentas estatísticas para determinar se um processo está sob controle, sob o ponto de vista estatístico [Lantzy, 1992]. Um processo está sob controle se seu comportamento, descrito por dados coletados para medidas de desempenho definidas para o processo, é estável, ou seja, se suas variações encontram-se dentro dos limites esperados, determinados com base em dados históricos. Um processo estável tem comportamento repetível, sendo possível prever seu desempenho em execuções futuras e, com isso, preparar planos que sejam alcançáveis e melhorá-lo continuamente. Por outro lado, um processo que apresenta variações que ultrapassam os limites esperados é instável e as 
causas dessas variações, chamadas causas especiais, precisam ser investigadas e tratadas através de ações de melhoria que visem à estabilização [Florac e Carleton, 1999].

Ter estabilizado seus processos é uma característica das organizações que buscam ou encontram-se nos mais elevados níveis de maturidade.

\section{Desenvolvimento do IABM}

A Figura 1 apresenta uma visão geral do processo de desenvolvimento do IABM, sendo seguida de sua descrição.

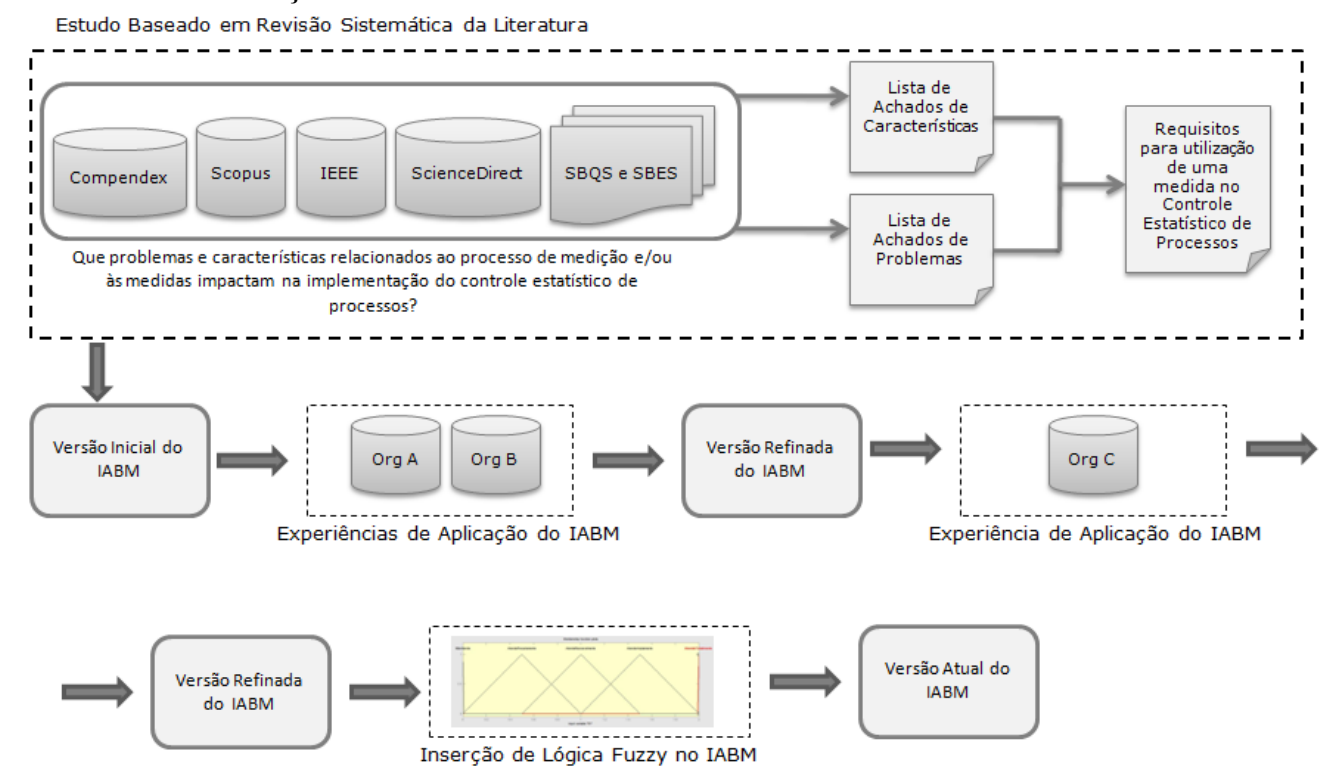

Figura 1. Desenvolvimento do IABM.

Para desenvolver o IABM, inicialmente foi realizado um estudo baseado em revisão sistemática da literatura a partir do qual foram obtidas duas listas de achados: uma de fatores relacionados às medidas e/ou à medição que influenciam positivamente na implementação do controle estatístico de processos de software (chamados de características no estudo) e uma de fatores que influenciam negativamente (chamados de problemas no estudo). A partir dessas listas de achados foi identificado um conjunto de requisitos considerados necessários para a aplicação de uma medida no controle estatístico de processos. A descrição completa do estudo baseado em revisão sistemática da literatura, com o detalhamento da obtenção das listas de achados e de requisitos encontra-se em [Barcellos, 2009].

O conjunto de requisitos identificado foi, então, utilizado para a criação da primeira versão do IABM, formada por um checklist para avaliar cada medida da base de medidas e os dados para ela coletados. Essa versão inicial foi avaliada através de experiências de aplicação em bases de medidas de duas organizações. O principal objetivo da avaliação da versão inicial foi verificar se os requisitos identificados eram adequados. Para isso, os principais questionamentos realizados foram:

(a) Uma medida que atende aos requisitos do IABM pode, realmente, ser aplicada no controle estatístico de processos de forma satisfatória?

(b) Uma medida que não atende aos requisitos do IABM é, realmente, não adequada ao controle estatístico de processos? 
Para responder a essas questões, as medidas foram submetidas à avaliação utilizando-se o checklist do IABM. Em seguida, os dados das medidas avaliadas foram aplicados em gráficos de controle. Os resultados obtidos com a aplicação dos dados das medidas em gráficos de controle foram ao encontro das avaliações realizadas pelo IABM. Ou seja, as medidas consideradas adequadas ao controle estatístico de processos segundo a avaliação pelo IABM puderam corretamente ser utilizadas nos gráficos de controle e forneceram informações sobre o desempenho dos processos, úteis aos objetivos da organização. Em contrapartida, as medidas que não foram consideradas adequadas ao controle estatístico de processos segundo a avaliação do IABM não puderam ser utilizadas nos gráficos de controle, ou, quando puderam, não foram capazes de descrever o desempenho dos processos e fornecer informações relevantes aos objetivos da organização. A versão inicial do IABM e os resultados de sua aplicação foram registrados em [Barcellos e Rocha, 2008b, a].

Apesar dos resultados das experiências iniciais de aplicação do IABM terem mostrado, considerando as bases avaliadas, que os requisitos identificados eram adequados, durante a aplicação do IABM percebeu-se que seria necessário fazer uma reestruturação em sua forma de aplicação, uma vez que, para avaliar as medidas propriamente ditas e os dados para elas coletados, foi necessário avaliar também o Plano de Medição e a estrutura da base de medidas. Assim, o IABM foi evoluído, passando a ser composto por quatro checklists: um para avaliação do Plano de Medição, um para avaliação da estrutura da base de medidas, um para avaliação das medidas definidas e um para avaliação dos dados coletados para as medidas. Além dessa alteração, na segunda versão do IABM foram formalizados os procedimentos de avaliação de cada requisito, bem como ações corretivas possíveis quando um requisito não for atendido.

A segunda versão do IABM foi, então, aplicada para avaliar a base de medidas de uma terceira organização. Essa experiência revelou, ainda, a necessidade de alguns pequenos ajustes no IABM. Os ajustes identificados foram considerados simples, uma vez que estavam relacionados ao texto do IABM, buscando tornar seu entendimento mais claro.

Finalmente, após essa experiência de aplicação do IABM, considerando que a avaliação de uma base de medidas tem caráter subjetivo, foram incluídos no IABM alguns princípios da Lógica Fuzzy para determinar quão adequada ao controle estatístico de processos é uma base de medidas. Essa alteração resultou na versão atual do IABM, que é apresentada na próxima seção.

\section{Instrumento para Avaliação de Bases de Medidas Considerando Adequação ao Controle Estatístico de Processos}

A avaliação de uma base de medidas utilizando-se o instrumento definido é composta pela avaliação de quatro itens: o Plano de Medição, a estrutura da base de medidas, as medidas propriamente ditas e os dados coletados para essas medidas. Além disso, uma vez que, de acordo com a abordagem de melhoria de processos de software para a alta maturidade, somente os processos considerados críticos para a organização devem ser submetidos ao controle estatístico de processos, é desejável que a organização identifique esses processos antes da avaliação da base de medidas, a fim de evitar a 
avaliação desnecessária de medidas não relacionadas a esses processos ou a tendência à escolha de processos que tenham medidas aplicáveis, porém que não sejam críticos.

A Figura 2 mostra uma visão geral do instrumento.

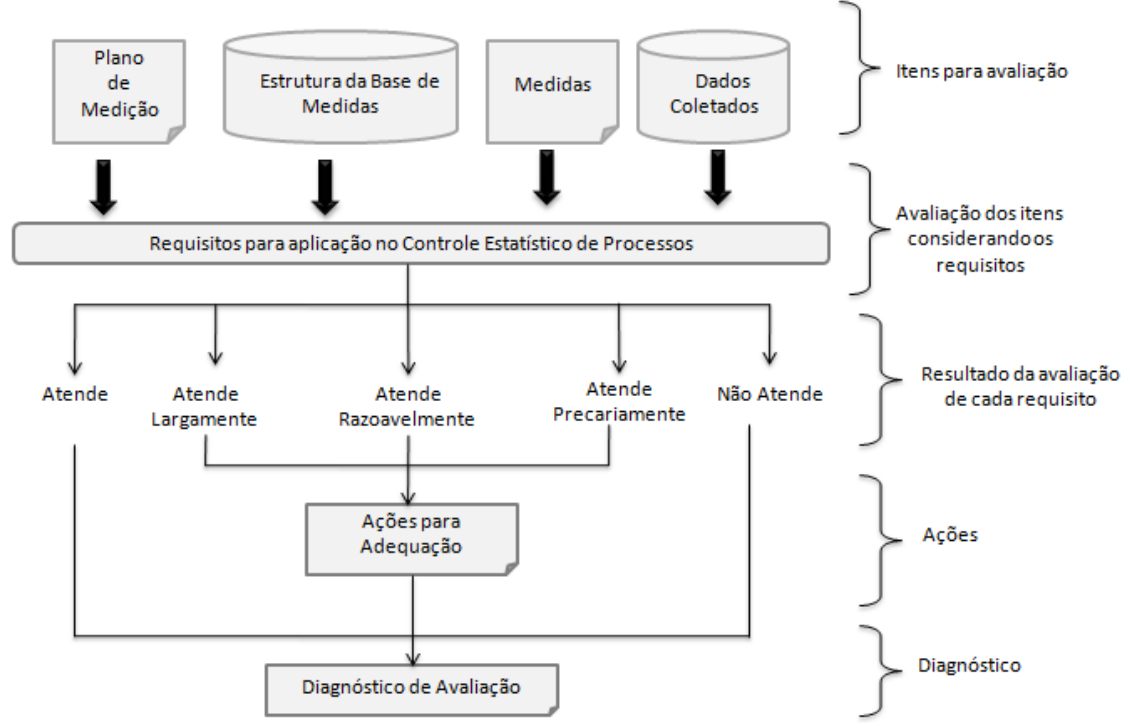

Figura 2. Visão geral do IABM.

Cada item considerado pelo instrumento é submetido à avaliação considerandose um conjunto de requisitos. A avaliação de cada item segundo cada requisito pode produzir um dos seguintes resultados:

(i) Atende: o item satisfaz totalmente o requisito e nenhuma ação de alteração do item avaliado é necessária em relação ao requisito considerado.

(ii) Atende Largamente, Atende Razoavelmente ou Atende Precariamente: o item não satisfaz o requisito, mas é possível realizar ações que irão adequálo a fim de satisfazer o requisito em questão e, consequentemente, permitir sua utilização no controle estatístico de processos. O grau de atendimento do item ao requisito (Largamente, Razoavelmente ou Precariamente) está diretamente relacionado com o esforço necessário para realizar as ações que levarão o item a atender o requisito em questão. Quanto mais esforço, menor o grau de atendimento.

(iii) Não Atende: o item não satisfaz o requisito e não há ações possíveis para adequar o item avaliado ao controle estatístico de processos, sendo necessário descartá-lo e redefini-lo, se pertinente.

Quando o resultado da avaliação de um requisito é Atende Largamente, Atende Razoavelmente ou Atende Precariamente, são sugeridas Ações para Adequação. Essas ações são orientações providas à organização que visam à realização de correções que permitam a utilização do item avaliado no controle estatístico de processos.

Os resultados da avaliação de uma base de medidas são registrados em um documento denominado Diagnóstico de Avaliação, que inclui, além da avaliação detalhada de cada item, sugestões das ações de adequação possíveis e o grau de adequação da base de medidas como um todo ao controle estatístico de processos, dado em percentual. 


\subsection{Checklists do IABM}

Nas figuras 3 a 6 são apresentados os checklists do IABM. Vale ressaltar que os checklists para avaliação do Plano de Medição (Figura 3) e da estrutura da base de medidas (Figura 4) são aplicados uma única vez durante uma avaliação de uma base de medidas, enquanto que os checklists para avaliação das medidas (Figura 5) e dos dados coletados (Figura 6) devem ser aplicados uma vez para cada medida avaliada.

\section{Item: Plano de Medição}

Legenda: $\mathrm{A}=$ Atende; $\mathrm{AL}=$ Atende Largamente; $\mathrm{AR}=$ Atende Razoavelmente; $\mathrm{AP}=$ Atende Precariamente; NA $=$ Não Atende, NFPA $=$ Não foi possivel avaliar

\begin{tabular}{|c|l|l|l|l|l|l|}
\hline \multicolumn{4}{|c|}{ Requisitos } & \multicolumn{3}{|c|}{ Avaliação } \\
\hline $\begin{array}{l}\text { 1. O Plano de Medição da Organização encontra-se alinhado aos objetivos da } \\
\text { organização. }\end{array}$ & ( ) A & ( ) AL & ( ) AR & ( ) AP & ( ) NA & ( ) NFPA \\
\hline $\begin{array}{c}\text { 1.1 Os objetivos de negócio da organização relevantes à medição estão } \\
\text { registrados no Plano de Medição. }\end{array}$ & ( ) A & ( ) AL & ( ) AR & ( ) AP & ( ) NA & ( ) NFPA \\
\hline $\begin{array}{c}1.2 \text { Os objetivos de medição estão registrados no Plano de Medição e } \\
\text { corretamente associados aos objetivos de negócio da organização. }\end{array}$ & ( ) A & ( ) AL & ( ) AR & ( ) AP & ( ) NA & ( ) NFPA \\
\hline $\begin{array}{c}1.3 \text { As necessidades de informação para monitoramento dos objetivos } \\
\text { de medição estão identificadas. }\end{array}$ & ( ) A & ( ) AL & ( ) AR & ( ) AP & ( ) NA & ( ) NFPA \\
\hline $\begin{array}{c}\text { 1.4 As medidas capazes de fornecer as informaçães necessárias ao } \\
\text { monitoramento dos objetivos de medição estão identificadas e } \\
\text { devidamente associadas. }\end{array}$ & ( ) A & ( ) AL & ( ) AR & ( ) AP & ( ) NA & ( ) NFPA \\
\hline
\end{tabular}

Figura 3. Checklist para avaliação do Plano de Medição.

\section{Item: Estrutura da Base de Medidas}

Legenda: $\mathrm{A}$ = Atende; $\mathrm{AL}=$ Atende Largamente; $\mathrm{AR}=$ Atende Razoavelmente; $\mathrm{AP}=$ Atende Precariamente; NA = Não Atende, NFPA = Não foi possível avaliar

\begin{tabular}{|c|c|c|c|c|c|c|}
\hline Requisitos & \multicolumn{6}{|c|}{ Avaliação } \\
\hline $\begin{array}{l}\text { 1. A base de medidas apresenta-se bem estruturada e permite que as } \\
\text { medidas sejam integradas aos processos e atividades da organização. }\end{array}$ & ( ) A & ( ) AL & ( ) AR & ( ) AP & ( ) NA & ( ) NFPA \\
\hline $\begin{array}{l}1.1 \text { A estrutura definida para a base de medidas permite relacionar as } \\
\text { medidas definidas aos processos e atividades da organização nos quais } \\
\text { a medição deve ser realizada. }\end{array}$ & ( ) A & ( ) $\mathrm{AL}$ & ( ) AR & ( ) AP & ( ) NA & ( ) NFPA \\
\hline $\begin{array}{l}1.2 \mathrm{~A} \text { base de medidas é única ou composta por diversas fontes } \\
\text { corretamente integradas. }\end{array}$ & ( ) A & ( ) AL & ( ) AR & ( ) AP & ( ) NA & ( ) NFPA \\
\hline 2. Os projetos são caracterizados satisfatoriamente. & ( ) A & ( ) $\mathrm{AL}$ & ( ) AR & ( ) AP & ( ) NA & ( ) NFPA \\
\hline $\begin{array}{l}\text { 3. Um mecanismo de identificação de similaridade entre projetos é } \\
\text { estabelecido. }\end{array}$ & ( ) A & ( ) AL & ( ) AR & ( ) AP & ( ) NA & ( ) NFPA \\
\hline 4. É possível identificar a versão dos processos executados nos projetos. & ( ) A & ( ) AL & ( ) AR & ( ) AP & ( ) NA & ( ) NFPA \\
\hline $\begin{array}{l}\text { 5. É possível armazenar e recuperar as informações de contexto das } \\
\text { medidas coletadas. }\end{array}$ & ( ) A & ( ) $\mathrm{AL}$ & ( ) AR & ( ) AP & ( ) NA & ( ) NFPA \\
\hline \multicolumn{7}{|l|}{ Para cada medida coletada, é possível armazenar e recuperar: } \\
\hline $\begin{array}{l}5.1 \text { Momento da medição (processo e atividade nos quais a medição foi } \\
\text { realizada) }\end{array}$ & ( ) A & ( ) AL & ( ) AR & ( ) AP & ( ) NA & ( ) NFPA \\
\hline $\begin{array}{l}5.2 \text { Condições da medição (dados relevantes sobre a execução do } \\
\text { processo ou projeto no momento da coleta da medida). }\end{array}$ & ( ) A & ( ) $\mathrm{AL}$ & ( ) AR & ( ) AP & ( ) NA & ( ) NFPA \\
\hline 5.3 Executor da medição. & ( ) A & ( ) AL & ( ) AR & ( ) AP & ( ) NA & ( ) NFPA \\
\hline 5.4 Projeto no qual a medida foi coletada. & ( ) A & ( ) AL & ( ) AR & ( ) AP & ( ) NA & ( ) NFPA \\
\hline 5.5 Características do projeto no qual a medida foi coletada. & ( ) A & ( ) $\mathrm{AL}$ & ( ) AR & ( ) AP & ( ) NA & ( ) NFPA \\
\hline
\end{tabular}

Figura 4. Checklist para avaliação da estrutura da base de medidas.

Cada requisito presente nos checklists do IABM possui uma descrição. Por exemplo, o requisito "Os projetos são caracterizados satisfatoriamente" (requisito 2 
do checklist de avaliação da estrutura da base de medidas - Figura 4) possui a seguinte descrição: Os projetos da organização devem ser caracterizados para permitir a identificação de projetos cujos dados coletados para as medidas possam ser analisados em conjunto ou sejam passíveis de comparações entre si. Uma caracterização é considerada satisfatória quando os subconjuntos formados pelos projetos que possuem o mesmo perfil, ou seja, cujos critérios de caracterização possuem os mesmos valores, são homogêneos. Exemplos de critérios para caracterizar projetos são: domínio do software, tipo de software, tecnologias envolvidas, restrições estabelecidas etc.

\begin{tabular}{|c|c|c|c|c|c|c|}
\hline \multicolumn{7}{|c|}{$\begin{array}{l}\text { Item: Medida } \\
\text { Legenda: } A=\text { Atende; } A L=\text { Atende Largamente; } A R=\text { atende Razoavelmente; } A P=\text { Atende Precariamente; } N A=\text { Não Atende, NFPA = Não foi possível } \\
\text { avaliar }\end{array}$} \\
\hline \multirow{2}{*}{$\begin{array}{l}\text { Requisitos } \\
\text { 1. A definição }\end{array}$} & \multicolumn{6}{|c|}{ Avaliação } \\
\hline & ( ) A & ( ) AL & ( ) AR & ( ) AP & ( ) NA & ( ) NFPA \\
\hline \multicolumn{7}{|l|}{ A definição operacional da medida inclui corretamente: } \\
\hline 1.1 Definição da medida. & ( ) A & ( ) $\mathrm{AL}$ & ( ) $A R$ & ( ) AP & ( ) NA & ( ) NFPA \\
\hline 1.2 Entidade medida. & ( ) A & ( ) AL & ( ) $A R$ & ( ) AP & ( ) NA & ( ) NFPA \\
\hline 1.3 Propriedade medida. & ( ) A & ( ) $\mathrm{AL}$ & ( ) AR & ( ) AP & ( ) NA & ( ) NFPA \\
\hline 1.4 Unidade de medida. & ( ) A & ( ) AL & ( ) AR & ( ) AP & ( ) NA & ( ) NFPA \\
\hline 1.5 Tipo de escala. & ( ) A & ( ) AL & ( ) AR & ( ) AP & ( ) NA & ( ) NFPA \\
\hline 1.6 Valores da escala. & ( ) A & ( ) $\mathrm{AL}$ & ( ) $A R$ & ( ) AP & ( ) NA & ( ) NFPA \\
\hline 1.7 Intervalo esperado dos dados. & ( ) A & ( ) $\mathrm{AL}$ & ( ) $A R$ & ( ) AP & ( ) NA & ( ) NFPA \\
\hline 1.8 Fórmula(s) (se aplicável). & ( ) A & ( ) AL & ( ) AR & ( ) AP & ( ) NA & ( ) NFPA \\
\hline 1.9 Descrição precisa do procedimento de medição. & ( ) A & ( ) AL & ( ) AR & ( ) AP & ( ) NA & ( ) NFPA \\
\hline 1.10 Responsável pela medição. & ( ) A & ( ) AL & ( ) AR & ( ) AP & ( ) NA & ( ) NFPA \\
\hline 1.11 Momento da medição. & ( ) A & ( ) AL & ( ) AR & ( ) AP & ( ) NA & ( ) NFPA \\
\hline 1.12 Periodicidade de Medição. & ( ) A & ( ) AL & ( ) AR & ( ) AP & ( ) NA & ( ) NFPA \\
\hline $\begin{array}{l}1.13 \text { Descrição precisa do procedimento de análise (se } \\
\text { indispensável). }\end{array}$ & ( ) A & ( ) AL & ( ) $A R$ & ( ) AP & ( ) NA & ( ) NFPA \\
\hline 1.14 Periodicidade da análise (se aplicável). & ( ) A & ( ) AL & ( ) AR & ( ) AP & ( ) NA & ( ) NFPA \\
\hline 2. A medida está alinhada a objetivos dos projetos ou da organização. & ( ) A & ( ) AL & ( ) AR & ( ) AP & ( ) NA & ( ) NFPA \\
\hline \multicolumn{7}{|l|}{ A medida está associada a: } \\
\hline 2.1 Objetivo da organização. & ( ) A & ( ) $\mathrm{AL}$ & ( ) AR & ( ) AP & ( ) NA & ( ) NFPA \\
\hline 2.2 Objetivo dos projetos. & ( ) A & ( ) AL & ( ) AR & ( ) AP & ( ) NA & ( ) NFPA \\
\hline $\begin{array}{l}\text { 3. Os resultados da análise da medida são relevantes às tomadas de } \\
\text { decisão. }\end{array}$ & ( ) A & & & & ( ) NA & ( ) NFPA \\
\hline 4. Os resultados da análise da medida são úteis à melhoria de processo. & ( ) A & & & & ( ) NA & ( ) NFPA \\
\hline 5. A medida está relacionada ao desempenho de um processo. & ( ) A & & & & ( ) NA & ( ) NFPA \\
\hline 6. A medida está relacionada a um processo crítico. & ( ) A & & & & ( ) NA & ( ) NFPA \\
\hline $\begin{array}{l}\text { 7. A medida está associada a uma atividade ou processo que produz } \\
\text { item mensurável. }\end{array}$ & ( ) A & & & & ( ) NA & ( ) NFPA \\
\hline 8. As medidas correlatas à medida estão definidas. & $($ ) A & ( ) AL & ( ) AR & ( ) AP & ( ) NA & ( ) NFPA \\
\hline 9. As medidas correlatas à medida são válidas. & ( ) A & ( ) $\mathrm{AL}$ & ( ) AR & ( ) AP & ( ) NA & ( ) NFPA \\
\hline 10. A medida possui baixa granularidade. & ( ) A & ( ) AL & ( ) AR & ( ) AP & ( ) NA & ( ) NFPA \\
\hline 11. A medida é passível de normalização (se aplicável). & () $\mathrm{A}$ & ( ) AL & ( ) AR & ( ) AP & ( ) NA & ( ) NFPA \\
\hline 12. A medida está normalizada corretamente (se aplicável). & ( ) A & ( ) $\mathrm{AL}$ & ( ) $A R$ & ( ) AP & ( ) NA & ( ) NFPA \\
\hline $\begin{array}{l}\text { 13. Os critérios de agrupamento de dados para análise da medida estão } \\
\text { definidos. }\end{array}$ & $($ ) A & ( ) AL & ( ) AR & ( ) AP & ( ) NA & ( ) NFPA \\
\hline 14. A medida não considera dados agregados. & ( ) A & ( ) AL & ( ) AR & ( ) AP & ( ) NA & ( ) NFPA \\
\hline
\end{tabular}

Figura 5. Checklist para avaliação das medidas de software. 


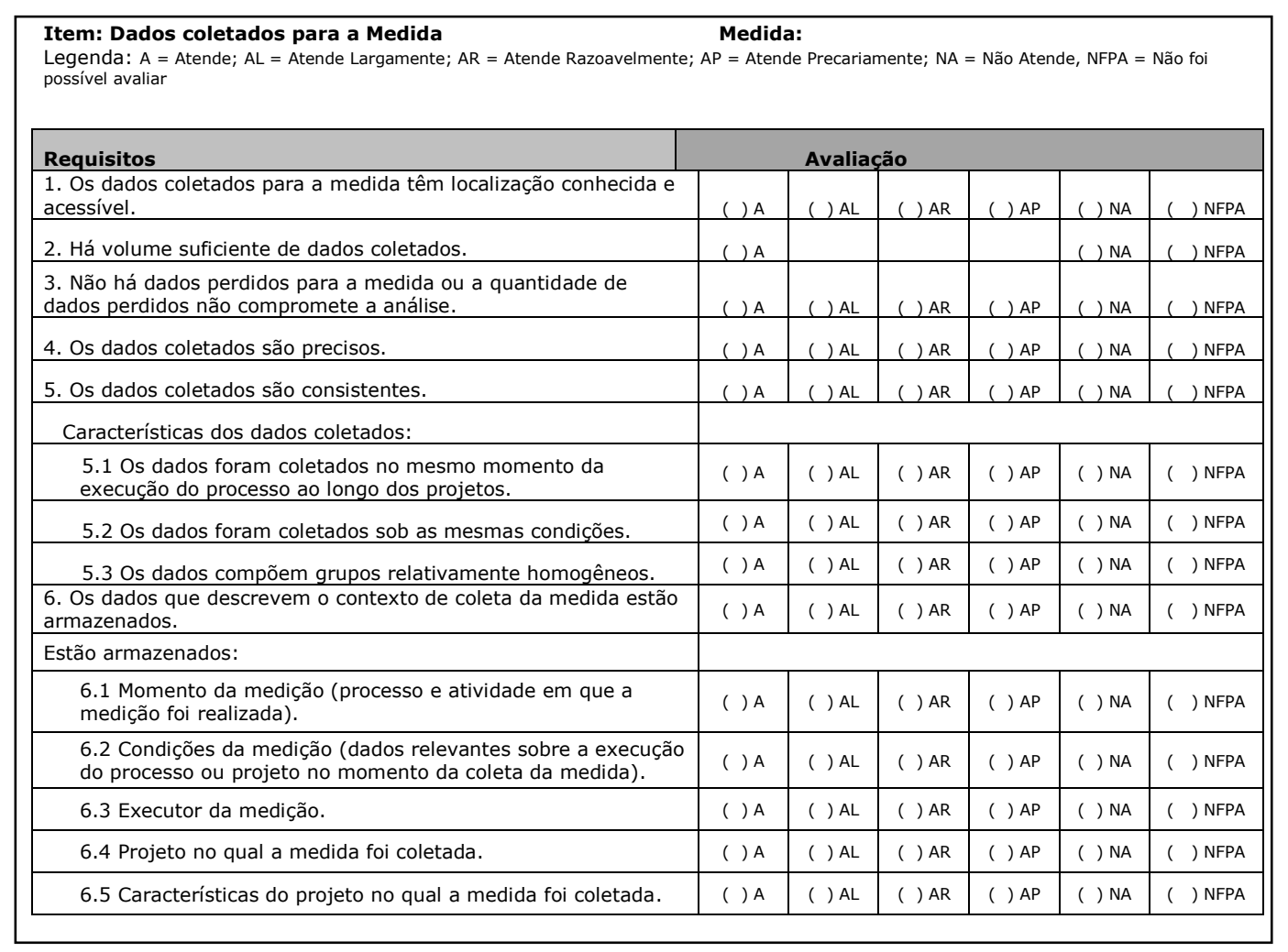

Figura 6. Checklist para avaliação dos dados coletados para as medidas de software.

\subsection{Avaliação do Atendimento aos Requisitos do IABM}

Conforme descrito anteriormente, a avaliação de cada requisito presente nos checklists do IABM produz um dos seguintes resultados: Atende, Atende Largamente, Atende Razoavelmente, Atende Precariamente ou Não Atende.

Observando os checklists apresentados na subseção anterior, é possível perceber que alguns requisitos só podem ter como resultados de avaliação Atende ou Não Atende. Para esses requisitos, não existe a possibilidade de atendimento parcial, uma vez que não existem ações de adequação que possam ser realizadas. Por exemplo, o requisito "A medida está relacionada ao desempenho de um processo" (requisito 5 do checklist de avaliação das medidas - Figura 5). Não há nada que possa ser feito para que uma medida que não descreve o desempenho de um processo seja adequadamente utilizada no controle estatístico de processos.

Para orientar a avaliação dos itens considerados pelo IABM, para cada requisito presente nos checklists para avaliação, foram descritas características que identificam cada um dos resultados de avaliação possíveis.

Como exemplo, a seguir é apresentada a descrição realizada para a avaliação do requisito "Os projetos são caracterizados satisfatoriamente" (requisito 2 do checklist de avaliação da estrutura da base de medidas - Figura 4).

Atende: A caracterização dos projetos é explícita, ou seja, há uma caracterização formalmente definida e implementada na estrutura da base de medidas para os projetos, baseada em critérios relevantes que permitem à organização identificar os perfis de projetos que a mesma desenvolve. Os subconjuntos formados pelos 
projetos que possuem o mesmo perfil, ou seja, cujos critérios de caracterização possuem os mesmos valores, são homogêneos.

Atende Largamente: A caracterização dos projetos é explícita, porém precisa de alguns critérios complementares que podem ser identificados analisando-se os dados dos projetos armazenados na base de medidas, realizando-se entrevistas com membros dos projetos ou analisando-se documentos dos projetos.

Atende Razoavelmente: A caracterização dos projetos é explícita, porém precisa de vários critérios complementares que podem ser identificados analisando-se os dados dos projetos armazenados na base de medidas, realizando-se entrevistas com membros dos projetos ou analisando-se documentos dos projetos.

Atende Precariamente: A caracterização dos projetos é implícita, ou seja, não há caracterização formal para os projetos, mas é possível identificar uma caracterização analisando-se os dados dos projetos armazenados na base de medidas, realizando-se entrevistas com membros dos projetos ou analisando-se documentos dos projetos.

Não Atende: Não há caracterização explícita ou ela é insuficiente e não é possível identificar critérios para estabelecer a caracterização analisando-se os dados armazenados para os projetos na base de medidas, realizando-se entrevistas com membros dos projetos ou analisando-se documentos dos projetos.

Cabe notar que alguns requisitos presentes nos checklists do IABM são divididos em sub-requisitos. O resultado da avaliação desses requisitos é obtido através de uma agregação dos resultados de seus sub-requisitos. O procedimento para a obtenção do resultado de avaliação de requisitos que possuem sub-requisitos é descrito na subseção 4.4 .

\subsection{Ações para Adequação aos Requisitos do IABM}

Quando o atendimento de um item em relação a um requisito é avaliado como Atende Largamente, Atende Razoavelmente ou Atende Precariamente, ações para adequação são sugeridas para que a organização altere o item avaliado para satisfazer o referido requisito, sem que seja necessário descartar o item. Nesse sentido, para cada requisito presente no IABM foram identificadas ações para adequação consideradas pertinentes.

Para exemplificar, utilizando o mesmo requisito para o qual foram descritas as orientações para avaliação na subseção anterior (Os projetos são caracterizados satisfatoriamente), a seguir são apresentadas as ações para adequação identificadas.

\section{Inadequações e Ações para Adequação:}

1. Os projetos possuem caracterização implícita na base de medidas.

a) Explicitar a caracterização implícita, analisando-se os dados armazenados para os projetos na base de medidas. Para isso, deve-se identificar, entre os dados registrados na base de medidas para os projetos, aqueles que descrevem características para os projetos executados. Por exemplo: restrições do projeto, equipe do projeto, tecnologias utilizadas, paradigma de desenvolvimento, domínio da aplicação, tipo de projeto, tamanho do projeto etc. 
b) Reestruturar a base de medidas, se necessário, para explicitar em classes (ou tabelas) e atributos os critérios identificados que caracterizam os projetos.

c) Registrar os dados dos projetos adequadamente na base de medidas modificada.

2. Os projetos não possuem caracterização (implícita ou explícita) na base de medidas.

a) Estabelecer uma caracterização com base na análise de documentos ou entrevistas com pessoas relacionadas aos referidos projetos. Por exemplo, os gerentes dos projetos realizados podem fornecer características dos projetos (tecnologias utilizadas, paradigma de desenvolvimento utilizado, tipo dos projetos, restrições consideradas etc.).

b) Reestruturar a base de medidas para explicitar em classes (ou tabelas) e atributos os critérios identificados para caracterizar os projetos.

c) Registrar os dados dos projetos adequadamente na base de medidas modificada.

3. A caracterização explícita dos projetos precisa de critérios complementares.

a) Refinar a caracterização. O refinamento pode ser realizado identificando-se novos critérios através da execução das ações apresentadas nos itens 1 e 2 anteriores.

\subsection{Grau de Adequação de uma Base de Medidas ao Controle Estatístico de Processos}

Os resultados das avaliações de cada requisito do Plano de Medição, da estrutura da base de medidas, das medidas e dos dados coletados determinam a adequação de cada um desses itens ao controle estatístico de processos. De acordo com os resultados da avaliação, um item pode ser Adequado, Largamente Adequado, Razoavelmente Adequado, Precariamente Adequado ou Não Adequado. Com base na adequação de seus itens, a adequação da base de medidas ao controle estatístico de processos é determinada.

No entanto, se a avaliação de duas bases de medidas determina que ambas têm adequação Razoavelmente Adequada, é correto afirmar que elas têm realmente a mesma adequação ou uma pode ser "mais adequada" que a outra? Uma delas pode, por exemplo, ser $40 \%$ adequada ao controle estatístico de processos e a outra $50 \%$ ?

Para diminuir a subjetividade dos termos Largamente Adequada, Razoavelmente Adequada, Precariamente Adequada, o IABM determina, além do termo que indica a adequação de uma base de medidas ao controle estatístico de processos, o seu grau de adequação a esse contexto, dado em percentual. Organizações podem utilizar o grau de adequação de suas bases de medidas para decidirem por sua correção ou pelo desenvolvimento de uma nova base.

Para, a partir dos resultados subjetivos da avaliação dos requisitos dos itens avaliados pelo IABM, obter como saída um valor único e objetivo que caracterize o grau de adequação da base de medidas ao controle estatístico de processos foram utilizados os princípios da Lógica Fuzzy e dos Conjuntos Fuzzy. Para isso foram 
seguidas as etapas do raciocínio fuzzy, presentes nos sistemas fuzzy: fuzzificação, inferência fuzzy e defuzzicação [Klir e Folger, 1998].

Inicialmente foi realizada a fuzzificação dos valores de entrada e de saída. Ou seja, as variáveis linguísticas referentes às entradas e saídas foram identificadas, seus termos linguísticos foram determinados e as funções de pertinência correspondentes foram definidas.

Os valores de entrada são os resultados da avaliação de cada requisito do IABM e são representados pelos termos linguísticos Atende, Atende Largamente, Atende Razoavelmente, Atende Precariamente, Não Atende. Os valores de saída são os possíveis graus de adequação de um item ao controle estatístico de processos e são representados pelos termos linguísticos Adequado, Largamente Adequado, Razoavelmente Adequado, Precariamente Adequado, Não Adequado. Para cada entrada e saída foram determinadas funções de pertinência. Por exemplo, para o termo linguístico Atende Largamente foi determinada a função de pertinência $(2.0,3.0,4.0) \mathrm{e}$ para o termo linguístico Largamente Adequado foi determinada a função de pertinência $(50,75,100)$. Dada a limitação de espaço, para exemplificação, são apresentadas as funções de pertinência de apenas um valor de entrada e um valor de saída. A descrição completa do raciocínio fuzzy adotado encontra-se em [Barcellos, 2009].

Realizada a fuzzificação, foram definidas as regras para a inferência fuzzy. Dado o grande número de variáveis de entrada e a grande variedade de combinações possíveis, a definição de regras no formato tradicional SE-ENTÃO para cobrir todas as combinações de entradas possíveis mostrou-se inapropriada. Decidiu-se, então, pela utilização do operador $O W A_{M E D I A}$ (Ordered Weighted Average) [Yager, 1988] para realizar a agregação das variáveis fuzzy de entrada em um único valor fuzzy de saída. Para isso, a cada termo linguístico de entrada foi atribuída uma nota, sendo: 4 - Atende, 3 - Atende Largamente, 2 - Atende Razoavelmente, 1 - Atende Precariamente, 0 - Não Atende. $\mathrm{O}$ agregado resultante de vários termos linguísticos de entrada (lembrando que um termo linguístico de entrada é o resultado da avaliação de um requisito) é igual à média de suas notas.

Para a avaliação de cada item, além da utilização de $O W A_{M E D I A}$, a seguinte regra foi estabelecida: se a nota correspondente a algum termo linguístico de entrada for 0 , então o agregado é 0 . Isso significa que, caso algum requisito de algum item seja avaliado como Não Atende, o item avaliado será considerado Não Adequado ao controle estatístico. A justificativa para essa regra está no fato de que, quando um requisito é avaliado como Não Atende, significa que não existem ações possíveis para adequar o item avaliado ao controle estatístico de processos, logo, o mesmo deve ser descartado, mesmo que atenda aos demais requisitos. Ter um requisito avaliado como Não Atende significa que o item pode ser reconstruído, porém, não pode ser adequado. Por isso, o não atendimento a um único requisito torna o item Não Adequado.

Para determinar o agregado resultante da avaliação de um item, inicialmente deve ser determinado o resultado da avaliação dos requisitos que possuem subrequisitos, utilizando-se $O W A_{M E D I A}$ e considerando-se a regra definida anteriormente. A obtenção do resultado da avaliação de um requisito com base nos resultados de seus sub-requisitos é ilustrada na Figura 7. 


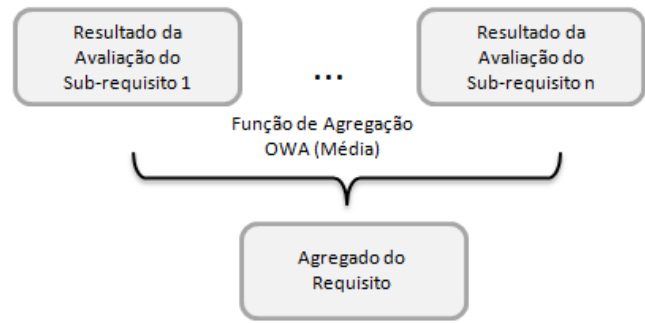

Figura 7. Avaliação de um requisito com sub-requisitos.

Determinados os resultados de avaliação dos requisitos com sub-requisitos, todos os resultados de avaliação dos requisitos do item são agregados aplicando-se $O W A_{M E D I A}$, para obter o agregado de cada item. A adequação da base de medidas como um todo é determinada pela média dos agregados dos itens avaliados.

Na Figura 8 é representado o procedimento adotado para determinar a adequação da base de medidas.
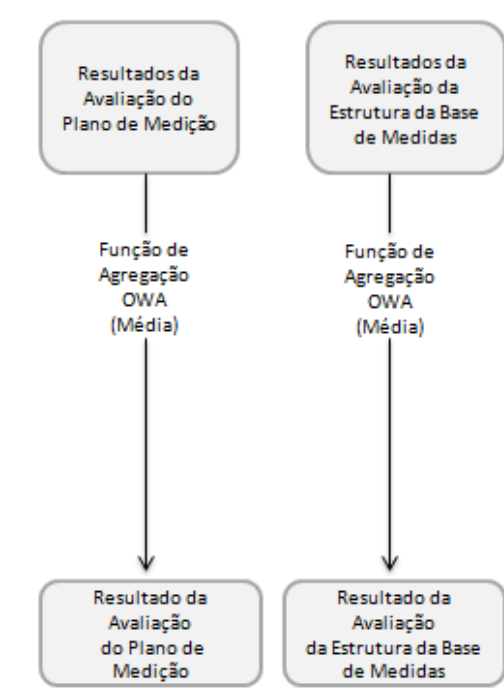
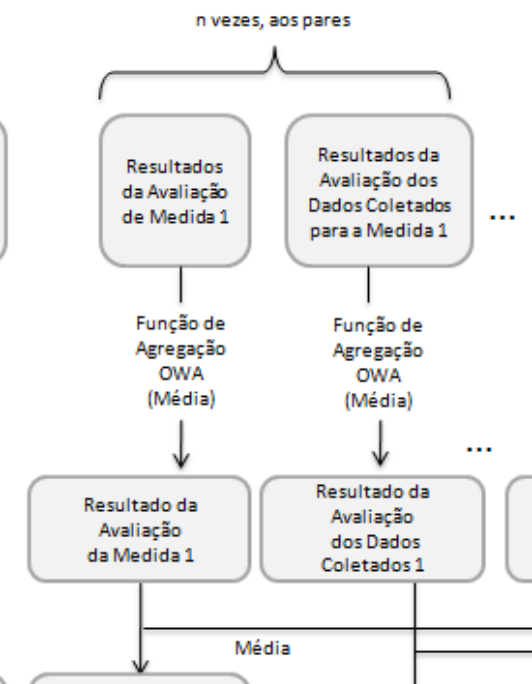

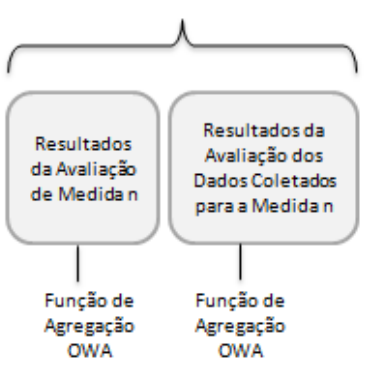

OWA OWA (Média) (Média)
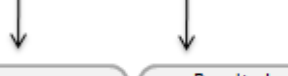
Resultado da Avaliação da Medidan Avaliaçã̃o dos Dados Coletados n

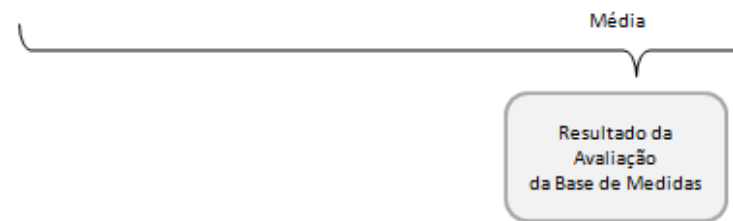

Figura 8. Procedimento adotado para obtenção do valor que determina a adequação

\section{da base de medidas.}

Obtida a saída fuzzy (Resultado da Avaliação da Base de Medidas), é utilizado o método de defuzzificação Centróide para determinar o valor crisp correspondente, ou seja, o valor único e objetivo equivalente ao grau de adequação da base de medidas. Vale notar que também é possível obter o grau de adequação de cada item, realizando a defuzzificação dos valores de seus resultados de avaliação. 
A implementação dos princípios da Lógica Fuzzy e dos Conjuntos Fuzzy para determinar a adequação de uma base de medidas a partir dos resultados de avaliação dos checklists do IABM foi realizada com apoio do software MATLAB R2007a ${ }^{1}$.

É importante ressaltar que a solução fuzzy adotada é uma solução inicial, não sendo considerada uma solução fuzzy ótima. No entanto, a solução proposta poderá ser refinada em oportunidade futura incluindo-se, por exemplo, pesos aos requisitos, determinados por especialistas e calibrados a partir de resultados de avaliações realizadas em bases de medidas.

\section{Trabalhos Correlatos}

Embora tenham sido encontrados vários trabalhos que destacam a necessidade de avaliação das medidas para que sejam aplicadas no controle estatístico de processos, apenas um [Tarhan e Demirors, 2006] inclui especificamente uma proposta para avaliação de medidas considerando sua utilidade no controle estatístico de processos. Nele os autores propõem uma abordagem para seleção dos processos que serão submetidos ao controle estatístico. Entre outros critérios relevantes para escolher os processos mais adequados, os autores incluem a existência de medidas úteis associadas aos processos. Para determinar se uma medida é útil, eles definem um conjunto de atributos para avaliação das medidas e dados coletados.

Apesar de incluir avaliação de medidas, até onde teve-se acesso, a abordagem proposta pelos autores é bastante limitada nesse contexto, já que seu foco é a seleção dos processos a serem submetidos ao controle estatístico de processos e não a avaliação das medidas. Os próprios autores afirmam que a análise da utilidade das medidas considerando apenas os atributos por eles definidos não é suficiente para selecionar as medidas mais adequadas ao controle estatístico de processos.

\section{Considerações Finais}

O crescente interesse das organizações de software em alcançar os níveis de maturidade mais elevados tem revelado algumas dificuldades características desses níveis, destacando-se a implementação do controle estatístico de processos.

A inexistência de medidas e dados adequados à aplicação das técnicas estatísticas que permitem que as organizações conheçam e melhorem o comportamento de seus processos tem se mostrado um obstáculo ao alcance da alta maturidade. Nesse contexto, este artigo apresentou um Instrumento para Avaliação de Bases de Medidas (IABM), elaborado com o propósito de auxiliar as organizações de software que possuem bases de medidas com dados coletados em projetos anteriores e que desejam realizar o controle estatístico de seus processos, a avaliarem se sua base de medidas está adequada ao controle estatístico e, caso não esteja, realizarem as adequações, quando possíveis.

Até o presente momento, o IABM foi utilizado para avaliar as bases de medidas de três organizações. Os resultados obtidos com as experiências de aplicação, apesar de não estarem explícitos neste artigo devido à limitação de espaço, permitem concluir que a avaliação e adequação das bases de medidas antes de realizar o controle estatístico de processos contribuem para que as organizações que possuem bases de medidas se

\footnotetext{
${ }^{1}$ Disponível em http://www.mathworks.com.
} 
preparem para iniciar o uso das técnicas estatísticas propriamente ditas, evitando que despendam esforço para implementar o controle estatístico de processos e, ao perceberem a inadequação de suas medidas, precisem interromper ou abandonar a implementação. Além disso, contribui para que as informações providas pelo controle estatístico de processos sejam realmente úteis, uma vez que as medidas e dados utilizados são adequados. Os resultados das experiências práticas de aplicação do IABM podem ser encontrados em [Barcellos e Rocha, 2008a, b] e [Barcellos, 2009].

Atualmente, o IABM não possui um software específico para apoiar sua utilização, sendo constituído basicamente por planilhas eletrônicas. Como trabalho futuro, planeja-se a implementação de uma ferramenta de apoio à utilização do IABM e a realização de novas experiências de aplicação.

\section{Agradecimentos}

Às organizações que participaram das experiências de aplicação do IABM e ao professor Geraldo Bonorino Xexéo, por sua colaboração na definição da solução fuzzy.

\section{Referências Bibliográficas}

BARCELLOS, M. P. (2009) "Uma Estratégia para Medição de Software e Avaliação de Bases de Medidas para Controle Estatístico de Processos em Organizações de Alta Maturidade", Tese de Doutorado, COPPE/UFRJ - Universidade Federal do Rio de Janeiro.

BARCELlOS, M. P., ROCHA, A. R. C. (2008a) "Avaliação de Bases de Medidas considerando sua Aplicabilidade ao Controle Estatístico de Processos de Software" Anais do VII Simpósio Brasileiro de Qualidade de Software (SBQS'08), Florianópolis - SC.

BARCEllos, M. P., ROCHA, A. R. C. (2008b) "Uma Abordagem de Apoio à Realização de Controle Estatístico de Processos de Software em Organizações de Alta Maturidade" XXXIV Conferência Latinoamericana de Informática (CLEI'08), Santa Fé - Argentina.

BOFFOLI, N., BRUNO, G., CAIVANO, D., MASTELlONI, G. (2008) "Statistical Process Control for Software: a Systematic Approach" In Proceedings of the 2008 ACM-IEEE International Symposium on Empirical Software Engineering and Measurement, Kaiserslautern - Germany, p. 327-329.

CARD, D., DOMZALSKI, K., DAVIES, G. (2008) "Making Statistics Part of Decision Making in an Engineering Organization" IEEE Software, v. 25, n. 3, p. 37-47.

CARD, D. N. (2004 ) "Statistical Techniques for Software Engineering Practice" In Proceedings of the 26th International Conference on Software Engineering ICSE'2004, Scotland, UK, p. 722-723.

CHRISSIS, M. B., KONRAD, M., SHRUM, S. (2006) "CMMI (Second Edition): Guidelines for Process Integration and Product Improvement", Addison-Wesley.

CURTIS, B., REIFER, D., SESHAGIRI, G. V., HIRMANPOUR, I., KEENI, G. (2008) "The Case for Quantitative Process Management" IEEE Software, v. 25, n. 3, p. 2428. 
FLORAC, W. A., CARLETON, A. D. (1999) " Measuring the Software Process: Statistical Process Control for Software Process Improvement", Addison Wesley.

GARCÍA, F., PIATTINI, M., RUIZ, F., CANFORA, G., VISAGGIO, C. A. (2004) "FMESP: Framework for the Modeling and Evaluation of Software Process" In Proceedings of the 2004 Workshop on Quantitative Techniques for Software Agile Process - QUTE-SWAP '04 - Newport Beach, California, p. 5-13.

GOU, L., WANG, Q., YUAN, J., YANG, Y., LI, M., JIANG, N. (2009) "Quantitative Defects Management in Interative Development with BiDefect" Software Process Improvement and Practice, v. 14, n. 4, p. 227-241

ISO/IEC (2003) "ISO/IEC 15504-2 - Information Technology - Software Process Assessment", International Organization for Standardization and the International Electrotechnical Commission, Geneva, Switzerland., International Organization for Standardization and the International Electrotechnical Commission, Geneva, Switzerland.

ISO/IEC (2008) "ISO/IEC 12207:2008 - Systems and Software Engineering - Software Life Cycle Process" International Organization for Standardization and the International Electrotechnical Commission, Geneva, Switzerland.

KITCHENHAM, B., JEFFERY, D. R., CONNAUNGHTON, C. (2007) "Misleading Metrics and Unsound Analyses" IEEE Software, v. 24, n. 2, p. 73 - 78.

KITCHENHAM, B., KUTAY, C., JEFFERY, R., CONNAUGHTON, C. (2006) "Lessons Learnt from the Analysis of Large-scale Corporate Databases" In Proceedings of the 28th International Conference on Software Engineering ICSE'06, Shanghai, China, p. 439-444.

KLIR, G. J., FOLGER, T. A. (1998) "Fuzzy Sets, Uncertainty and Information", Prentice Hall, New Jersey. .

LANTZY, M. A. (1992) "Application of Statistical Process Control to the Software Process" In Proceedings of the 9th Washington Ada Symposium on Empowering Software Users and Developers, ACM Press, p. 113-123.

SOFTEX (2009) "MPS.BR: Melhoria de Processo do Software Brasileiro - Guia Geral: 2009", Disponível em: http://www.softex.br/mpsbr.

TARHAN, A., DEMIRORS, O. (2006) "Investigating Suitability of Software Process and Metrics for Statistical Process Control" Lecture Notes in Computer Science, v. 4257, p. 88-99.

TARHAN, A., DEMIRORS, O. (2008) "Assessment of Software Process and Metrics to Support Quantitative Understanding" Lecture Notes in Computer Science, v. 4895, p. 102-113.

WANG, Q., GOU, L., JIANG, N., CHE, M., ZHANG, R., YANG, Y., LI, M. (2007) "An Empirical Study on Establishing Quantitative Management Model for Testing Process" Lecture Notes in Computer Science, v. 4470, p. 233-245.

WHEELER, D. J., POLING, R. S. (1998) "Building Continual Improvement: A Guide for Business", SPC Press.

YAGER, R. R. (1988) "On Ordered Weighted Averagingh Aaggregation Operators in Multicriteria Decision Making" IEEE Transactions on Systems, v. 18, n. 1, p. 183190. 